\title{
Ofatumumab: A Review in Relapsing Forms of Multiple Sclerosis
}

\author{
Connie Kang ${ }^{1} \cdot$ Hannah A. Blair ${ }^{1}$
}

Published online: 13 December 2021

(c) Springer Nature 2021, corrected publication 2021

\begin{abstract}
Ofatumumab (Kesimpta $\left.{ }^{\circledR}\right)$ is a fully human anti-CD20 monoclonal antibody that can be self-administered by patients and is approved in several countries worldwide for the treatment of relapsing forms of multiple sclerosis (MS). In two identical phase III trials in adults with relapsing forms of MS, subcutaneous ofatumumab was more effective than oral teriflunomide in reducing the annualized relapse rate, as well as reducing MRI-detected lesion activity and limiting worsening of disability. Ofatumumab had a generally manageable tolerability profile; the most common adverse events (AEs) included nasopharyngitis, headache, upper respiratory tract infections and urinary tract infections. AEs of special interest (AESIs) included infections and injection-related reactions, which were generally manageable. There was no apparent association between changes in immunoglobulin $\mathrm{G}$ or $\mathrm{M}$ levels and the risk of serious infections after 3.5 years of ofatumumab treatment. Thus, ofatumumab is a convenient treatment option that is effective and has a generally manageable tolerability profile in adults with relapsing forms of MS.

Plain Language Summary MS is an incurable disease that affects $\approx 2.8$ million people worldwide. Limiting the progression of disability associated with this disease is crucial, and treatments such as teriflunomide or monoclonal antibodies can prevent the relapses which define MS. Ofatumumab (Kesimpta ${ }^{\circledR}$ ), a monoclonal antibody, works by reducing the level of B cells which contribute to the development and progression of MS. Ofatumumab is approved in several countries worldwide to treat adults with certain relapsing forms of MS. It is administered by subcutaneous injection once per month and is the first therapy of its kind that patients can self-inject at home. In clinical trials, ofatumumab was more effective than teriflunomide in reducing the annual relapse rate, as well as slowing both the progression of disability and formation of new MS lesions in the brain. Ofatumumab had a generally manageable tolerability profile, although treatment resulted in infections and injection-related reactions; these were generally manageable with treatment. Thus, ofatumumab is an effective and convenient treatment option, with a generally manageable tolerability profile, in adults with relapsing forms of MS.
\end{abstract}

The manuscript was reviewed by: $Y$. Aladro, Department of Neurology, University Hospital of Getafe, Madrid, Spain; $\boldsymbol{S} . \boldsymbol{S}$. Bachhav, Clinical Pharmacology and Pharmacometrics, AbbVie Inc., North Chicago, IL, USA; J.L. Bainbridge, Department of Clinical Pharmacy, University of Colorado Anschutz Medical Campus, Aurora, CO, USA; S. de Biase, Neurology Unit, Ospedale dell'Angelo, Venezia-Mestre, Italy; M. Habek, Referral Center for Autonomic Nervous System Department of Neurology, School of Medicine, University of Zagreb, Zagreb, Croatia.

Connie Kang

demail@springer.com

1 Springer Nature, Mairangi Bay, Private Bag 65901, Auckland 0754, New Zealand
Digital Features for this Adis Drug Evaluation can be found at https://doi.org/10.6084/m9.figshare.16934563

Ofatumumab: clinical considerations in relapsing forms of multiple sclerosis

Convenient self-administered treatment regimen (oncemonthly subcutaneous injections)

Significantly reduces annualized relapse rate, MRIdetected lesion activity and disability progression compared with teriflunomide

Generally manageable tolerability profile; AESIs include infections and injection-related reactions 


\section{Introduction}

Multiple sclerosis (MS) is an incurable disease with a worldwide prevalence of $\approx 2.8$ million people, affecting up to three times more women than men [1]. While symptom management is important in MS, relapse prevention is crucial in patients with relapsing forms of MS (i.e. clinically isolated syndrome, relapsing-remitting MS and secondary progressive MS) [2, 3]. For these patients, many types of diseasemodifying treatments (DMTs) are effective in reducing the incidence of MS relapses [3]. Patients with MS consistently display increased antibody production through B cell activity [4]. Monoclonal antibodies are among the most effective DMTs, including those that target the CD20 molecule on $\mathrm{B}$ cell surfaces and cause B cell depletion, leading to less relapses and improvements in MS symptoms [4-6]. Choice of a particular DMT, however, is decided based on patientspecific factors, so any available DMT may be used as initial treatment [7].

Ofatumumab (Kesimpta ${ }^{\circledR}$ ), a recombinant human antiCD20 monoclonal immunoglobulin G1 antibody, was initially approved for the treatment of chronic lymphocytic leukaemia (Arzerra ${ }^{\circledR}$; previously reviewed [8]), and is now approved as a subcutaneous (SC) injection in several countries (including the USA [9], those in the EU [10] and Japan [11]) for the treatment of relapsing forms of MS. Ofatumumab is the first B-cell-targeting therapy that is intended for self-administration at home (following initial training by a healthcare professional). This article summarizes the efficacy and tolerability of ofatumumab in the treatment of relapsing forms of MS.

\section{Pharmacological Properties of Ofatumumab}

While the precise mechanism of action of ofatumumab is unknown, it is understood that the FAB portion of ofatumumab selectively binds to and inhibits CD20, a transmembrane phosphoprotein expressed on B lymphocytes [9, 10, 12]; ofatumumab binds to a region of the $C D 20$ different from that of other anti-CD20 antibodies [6]. This leads to B cell (and T cell) depletion via complement-mediated CD20+ $\mathrm{B}$ cell lysis and antibody-dependent cell-mediated cytotoxicity $[9,10,12]$. Key pharmacological properties of ofatumumab are summarized in Table $1[9,10]$.

In a 12-week, open-label, bioequivalence phase II study (APLIOS; $n=284$ ), treatment with SC ofatumumab $20 \mathrm{mg}$ (loading doses on days 1, 7 and 14, then monthly maintenance doses, thereafter) resulted in significant $B$ cell count reduction (Table 1) [13]. An anti-CD19+ B-cell assay was used as a surrogate to measure $\mathrm{CD} 20+\mathrm{B}$ cell count as ofatumumab interferes with the CD20 assay $[9,13]$.

In phase III trials (ASCLEPIOS I and II), B cell counts and serum levels of neurofilament light chain (NfL), a biomarker of neuroaxonal damage, were significantly reduced by ofatumumab therapy [6]. The overall serum NfL level was $13.3 \mathrm{pg} / \mathrm{mL}$ at baseline; after 24 months of treatment in both studies, the mean serum NfL level was significantly $(p<0.001)$ lower in ofatumumab recipients $(6.9 \mathrm{pg} / \mathrm{mL}$ and $6.8 \mathrm{pg} / \mathrm{mL}$ in ASCLEPIOS I and II, respectively) than in teriflunomide recipients $(9.0 \mathrm{pg} / \mathrm{mL}$ in each of ASCLEPIOS I and II) [6].

\section{Therapeutic Efficacy of Ofatumumab}

In phase II trials (APLIOS [13], APOLITOS [14] and MIRROR [15]), SC ofatumumab was associated with significant reductions in new brain lesion activity (from baseline or versus placebo), as measured by magnetic resonance imaging (MRI), in patients with relapsing forms of MS. Due to the availability of phase III data, the phase II trials are not discussed further.

Approval of SC ofatumumab in patients with relapsing forms of MS was based on the results from two identical, multicentre, double-blind, randomized, active-comparator controlled phase III trials, ASCLEPIOS I and II $(n=927$ and 955; clinical trial design is summarized in Fig. 1) [6, $9,10]$. A double-dummy design was used. In these trials, eligible patients were aged 18-55 years and had a diagnosis of MS with a relapsing-remitting course or a secondary progressive course with disease activity [6]. Other inclusion criteria were an Expanded Disability Status Scale (EDSS) score of 0-5.5 (range 0-10, where a higher score equals greater disability); $\geq 1$ relapse 1 year before screening, $\geq 2$ relapses 2 years before screening or $\geq 1$ lesion detected using gadolinium (Gd) enhancement on MRI in the year before randomization; and neurologically stable condition for 1 month before randomization. Exclusion criteria included MS diagnosis more than 10 years ago with EDSS score $\leq 2$, neuromyelitis optica, active infection or an immunocompromised state [6].

Patients received SC ofatumumab $20 \mathrm{mg}$ once monthly (following initial weekly doses at days 1, 7 and 14) or oral teriflunomide $14 \mathrm{mg}$ once daily for up to 30 months [6]. Randomization was stratified according to geographic region and subtype of MS. The primary efficacy endpoint was the annualized relapse rate, defined as the number of confirmed MS relapses per year according to prespecified criteria. Key secondary endpoints are shown in Table 2 [6].

Baseline demographics and disease characteristics were similar across treatment arms in both phase III trials [6]. Averaged across ASCLEPIOS I and II [6], the mean age 


\section{Table 1 Pharmacological properties of ofatumumab}

Pharmacodynamic properties

B cell depletion

In the phase II APLIOS study, B cell count $<10$ cells $/ \mu \mathrm{L}$ was seen in $84.6 \%$ of ofatumumab recipients at day 14 and sustained by $>95 \%$ of pts until end of study [13]

In phase III trials, rapid and sustained reduction in B cell count (using an anti-CD19+ assay) was seen in ofatumumab recipients (and across all pt subgroups); $94 \%$ of pts had $<10$ cells/ $\mu \mathrm{L}$ (LLN 40 cells $/ \mu \mathrm{L}$ ) at study week 4 and sustained for up to week 120 with continued treatment $[6,9,10]$. B cell count was below LLN in $77.0-78.8 \%$ of pts after one dose, in $99.3-99.5 \%$ of pts at week 12 , and in $\approx 92-97 \%$ of pts from week $12-120$ [9]

B cell repletion

Median time to B cell recovery (to LLN or baseline) post treatment discontinuation was 24.6 weeks in phase III clinical trials [10]; pharmacokinetic modelling and simulation data for B cell repletion estimated a median of 23-40 weeks $[9,10]$. Faster post-treatment B-cell repletion than several other anti-CD20 monoclonal antibodies [28]

Immunogenicity $\quad$ Incidence of anti-drug antibodies in phase III trials too low $(0.2 \%$ of pts $)$ for evaluation $[9,10]$

Pharmacokinetic properties $[9,10]$

Non-linear pharmacokinetics; clearance decreased over time (due to B cell depletion) [10]

Absorbed mainly via the lymphatic system; at steady state, once-monthly SC dosing of ofatumumab 20 mg results in a mean $\mathrm{AUC}_{\tau}$ of 483 $\mu \mathrm{g} \cdot \mathrm{h} / \mathrm{mL}$ and a mean $\mathrm{C}_{\max }$ of $1.43 \mu \mathrm{g} / \mathrm{mL}$

Estimated steady-state volume of distribution was $5.42 \mathrm{~L}$

Metabolised by ubiquitous proteolytic enzymes into small peptides and amino acids

Eliminated by target-mediated route (binds to B cells) and target-independent route (mediated by non-specific endocytosis, then intracellular catabolism); higher baseline B cell count results in more target-mediated elimination, leading to greater clearance, which causes B cell depletion; estimated clearance $0.34 \mathrm{~L} /$ day following B cell depletion [9]

Steady-state half-life $\approx 16$ days

Drug interactions and effect in pts with abnormal liver or kidney function have not been studied (dose modification not expected [10])

$A U C_{\tau}$ area under the plasma concentration-time curve over the dosing interval, $C_{\max }$ maximum drug concentration, $L L N$ lower limit of normal, $p t(s)$ patient(s), $S C$ subcutaneous

Patients with MS with a

relapsing-remitting course or secondary progressive course with disease activity assessed for eligibility $(n=2557)$

\begin{tabular}{|l|l|}
\hline Screening & Randomized Double-Blind Treatment \\
\hline \multirow{2}{*}{ ASCLEPIOS I } & Ofatumumab $20 \mathrm{mg}(n=465)$ \\
\hline ASCLEPIOS II & Comparator teriflunomide $14 \mathrm{mg}(n=462)$ \\
\hline & Ofatumumab $20 \mathrm{mg}(n=481)$ \\
\hline
\end{tabular}

Annualized relapse rate

Fig. 1 Trial design of the randomized, double-blind, multinational phase III ASCLEPIOS trials in adults with relapsing forms of multiple sclerosis [6], with further information available in Table 2. Efficacy results are reported in the animated figure (available online). $M S$ multiple sclerosis, $R R$ rate ratio

was 38 years and $68 \%$ of patients were female. The majority $(89 \%)$ of patients were white [9]. Most (94\%) patients had relapsing-remitting MS and $40 \%$ had not received any previous DMT [6]. The mean number of Gd-enhancing T1 lesions per MRI scan was 1.5 and $61 \%$ of patients had no Gd-enhancing lesions. At baseline, the mean EDSS score was 2.9 and the normalized brain volume was $1442 \mathrm{~cm}^{3}$ [6].
Ofatumumab was more effective than teriflunomide at reducing relapse rates in adults with relapsing forms of MS. In the intent-to-treat (ITT) populations in both trials (median duration of treatment was 85 weeks), the annualized relapse rates were significantly lower in the ofatumumab groups than the teriflunomide groups (Table 2) [6, 9, 10]. The relative risk of a relapse was reduced by more than $50 \%$ in both trials $[6,9,10]$. 
Table 2 Efficacy of subcutaneous ofatumumab $20 \mathrm{mg}$ versus oral teriflunomide $14 \mathrm{mg}$ in patients with relapsing forms of multiple sclerosis in two phase III trials [6]

\begin{tabular}{|c|c|c|c|c|c|c|}
\hline \multirow[t]{2}{*}{ Endpoints } & \multicolumn{3}{|c|}{ ASCLEPIOS I } & \multicolumn{3}{|c|}{ ASCLEPIOS II } \\
\hline & OFA & TER & $\mathrm{RR} / \mathrm{TD}^{\mathrm{a}}(95 \% \mathrm{CI})$ & OFA & TER & $\mathrm{RR} / \mathrm{TD}^{\mathrm{a}}(95 \% \mathrm{CI})$ \\
\hline Annualized relapse rate ${ }^{\mathrm{b}}$ & 0.11 & 0.22 & $0.49(0.37-0.65)^{* * *}$ & 0.10 & 0.25 & $0.42(0.31-0.56)^{* * *}$ \\
\hline \multicolumn{7}{|l|}{ MRI-related endpoints ${ }^{c}$} \\
\hline Mean no. of Gd-enhancing T1 lesions per MRI scan ${ }^{\mathrm{d}}$ & 0.01 & 0.45 & $0.03(0.01-0.05)^{* * *}$ & 0.03 & 0.51 & $0.06(0.04-0.10)^{* * *}$ \\
\hline Annualized rate of new or enlarging $\mathrm{T} 2$ lesions $^{\mathrm{e}}$ & 0.72 & 4.00 & $0.18(0.15-0.22)^{* * *}$ & 0.64 & 4.15 & $0.15(0.13-0.19)^{* * *}$ \\
\hline Annual rate of loss in brain volume $(\%)^{\mathrm{f}}$ & 0.28 & 0.35 & $0.07(-0.02$ to 0.15$)$ & 0.29 & 0.35 & $0.07(-0.02$ to 0.15$)$ \\
\hline \multicolumn{7}{|l|}{ Disability-related endpoints ${ }^{\mathrm{g}}$} \\
\hline Confirmed disability worsening at 3 months $^{\mathrm{h}}$ (\% of pts) & \multicolumn{6}{|c|}{$10.9 \%$ OFA vs $15.0 \%$ TER [HR $0.66(95 \% \text { CI } 0.50-0.86)^{* *}$ ] } \\
\hline Confirmed disability worsening at 6 months $^{\mathrm{h}}$ (\% of pts) & \multicolumn{6}{|c|}{$8.1 \%$ OFA vs $12.0 \%$ TER [HR $0.68(95 \% \text { CI } 0.50-0.92)^{*}$ ] } \\
\hline Confirmed disability improvement at 6 months $\mathrm{s}^{\mathrm{i}}(\%$ of pts) & \multicolumn{6}{|c|}{$11.0 \%$ OFA vs $8.1 \%$ TER [HR 1.35 (95\% CI 0.95-1.92)] } \\
\hline
\end{tabular}

EDSS Expanded Disability Status Scale, $G d$ gadolinium, $H R$ hazard ratio, MRI magnetic resonance imaging, $O F A$ ofatumumab, pts patients, $R R$ rate ratio, TER teriflunomide, $T D$ treatment difference

$* p=0.01, * * p=0.002, * * * p<0.001$

${ }^{\mathrm{a}} \mathrm{RR}$ for primary endpoint and first two MRI-related endpoints, and TD for annual rate of loss in brain volume

${ }^{\text {b}}$ Primary efficacy endpoint (assessed in 454 and 452 pts in the OFA and TER groups in ASCLEPIOS I, and 469 pts in both treatment groups in ASCLEPIOS II)

${ }^{c}$ Endpoints evaluated hierarchically in order shown (provided all prior null hypotheses were rejected)

${ }^{\mathrm{d}}$ Assessed in 432 and 422 pts in the OFA and TER groups in ASCLEPIOS I, and 439 and 469 pts, respectively, in ASCLEPIOS II

${ }^{\mathrm{e}}$ Evaluated at end of study (assessed in 440 and 431 pts in the OFA and TER groups in ASCLEPIOS I, and 448 and 443 pts, respectively, in ASCLEPIOS II)

${ }^{\mathrm{f}}$ Assessed in 418 and 409 pts in the OFA and TER groups in ASCLEPIOS I, and 437 and 434 pts, respectively, in ASCLEPIOS II

${ }^{\mathrm{g}}$ Kaplan-Meier estimates at 24 months, based on prespecified meta-analyses using pooled data from both trials $(n=944$ and 931 , respectively, for disability worsening endpoints, and $n=749$ and 723 for disability improvement endpoint), and only if the null hypothesis for the primary endpoint was rejected

${ }^{\mathrm{h}}$ Disability worsening defined as an increase in EDSS score of $\geq 1.5,1.0$ or 0.5 points in pts with baseline EDSS score of $0,1-5$ or $\geq 5$, respectively

${ }^{\mathrm{i}}$ Disability improvement defined as a decrease in EDSS score of $\geq 1.0$ or 0.5 points if baseline EDSS score was 2.0-6.0 or 6.5-9.0 (sustained for at least 6 months)

For most secondary endpoints, ofatumumab was more effective than teriflunomide, significantly reducing the number of MRI-detected lesions and the proportion of patients with confirmed disability worsening at 3 and 6 months (Table 2) [6]. There were no statistically significant betweengroup differences for the annual rate of loss in brain volume ( $p \geq 0.12$ ) or the proportion of patients with a confirmed disability improvement at 6 months ( $p=0.09$ in the pooled trials) [6]. A similar effect of ofatumumab on the key disability- and MRI-related endpoint results compared to teriflunomide was observed across the exploratory subgroups analysed (age, sex, body weight, baseline disease and disability characteristics, previous non-steroid treatment for MS) [9, 10].

A post hoc analysis of the ASCLEPIOS trials evaluated the efficacy of ofatumumab in a subgroup of treatment-naïve patients who had been diagnosed with relapsing forms of MS within the last 3 years $(n=615)$ [16]. Baseline patient characteristics were consistent with those of patients with early MS. Compared with teriflunomide, ofatumumab therapy showed superior efficacy in primary and several key secondary efficacy endpoints. These reductions in disability- and MRI-related outcomes were comparable to those in the overall population [16]. Clinically relevant differences in the annualized relapse rate were not seen in patients regardless of baseline demographics or patient disease characteristics $[17,18]$.

\section{Tolerability of Ofatumumab}

SC ofatumumab $20 \mathrm{mg}$ had a generally manageable tolerability profile in patients with relapsing forms of $\mathrm{MS}$, as evaluated using pooled safety population data from ASCLEPIOS I and II [6, 9, 10]. Results were consistent with phase II trial data, in which SC ofatumumab was well tolerated in patients with relapsing forms of MS [14, 15, 19].

In the phase III trials, adverse events (AEs) were reported in $83.6 \%$ of ofatumumab recipients (791 of 946) and $84.2 \%$ of teriflunomide recipients (788 of 936) [6]. The most 


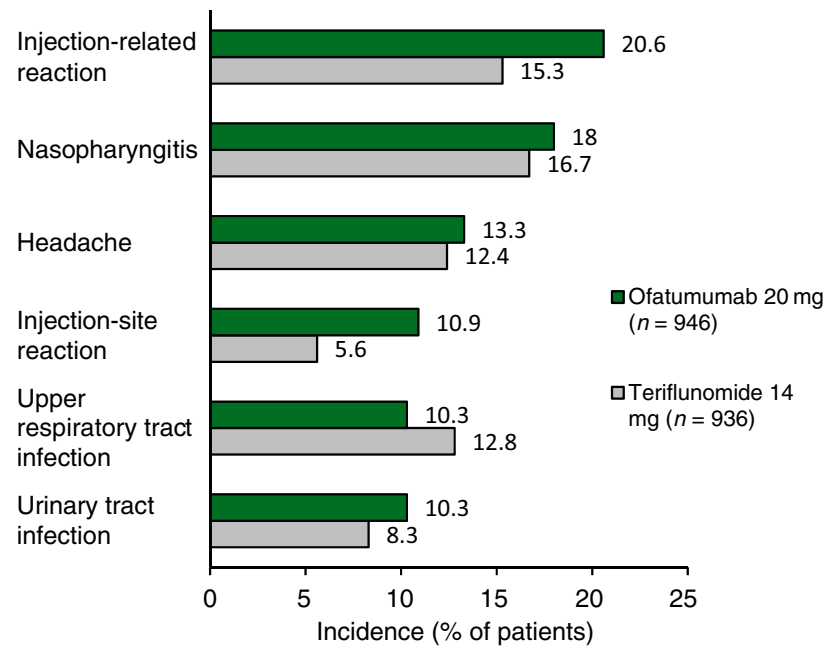

Fig. 2 Most common ( $\geq 10 \%$ of ofatumumab recipients) treatmentemergent adverse events in the safety population of two identical phase III trials (ASCLEPIOS I and II) in patients with relapsing forms of multiple sclerosis [20].

commonly reported AEs ( $\geq 10 \%$ of ofatumumab recipients) were injection-related reactions, nasopharyngitis, headache, injection-site reactions, upper respiratory tract infections and urinary tract infections (Fig. 2) [6]. Local injection-site reactions were most commonly ( $\geq 2 \%$ of patients) erythema, pain, itching and swelling, and were mild to moderate in severity $[9,10]$. Prophylaxis with medications such as pain relievers, antihistamines and/or steroids did not significantly reduce the frequency of these reactions [6]. In a post hoc analysis of ofatumumab in newly-diagnosed, treatment-naïve patients, the tolerability profile was consistent with that of the overall populations in ASCLEPIOS I and II [16].

Serious AEs were reported in $9.1 \%$ of ofatumumab recipients and $7.9 \%$ of teriflunomide recipients [6], and were most commonly (incidence $\geq 1 \%$ of patients) infections and infestations ( $2.5 \%$ vs $1.8 \%)$, injury, poisoning and procedural complications ( $1.4 \%$ vs $1.0 \%)$, psychiatric disorders $(1.1 \%$ vs $0.2 \%$ ) and neoplasms (1.0\% vs $0.4 \%$ ) [20]. Discontinuation of treatment due to AEs occurred in $5.7 \%$ and $5.2 \%$ of ofatumumab and teriflunomide recipients [10]; this was most commonly (3.3\%) due to low immunoglobulin M (IgM) levels (defined as $10 \%$ below the lower limit of normal) in ofatumumab recipients [9].

The immunogenicity of ofatumumab was assessed in ASCLEPIOS I and II $[9,10]$ and in an open-label, extension phase IIIb trial (ALITHIOS; $n=1703$ ) [21]. In the ASCLEPIOS trials, the mean IgM level was reduced by $30.9 \%$ after 48 weeks on-study, and by $38.8 \%$ after 96 weeks [10]. The IgM level was below $0.34 \mathrm{~g} / \mathrm{dL}$ in $14.3 \%$ of patients. The reduced IgM levels were not associated with an increased risk of infection or serious infection. Conversely, immunoglobulin $\mathrm{G}$ ( $\mathrm{IgG}$ ) levels were increased by $4.3 \%$ after 48 weeks, and by $2.2 \%$ after 96 weeks $[9,10]$.
The ALITHIOS trial assessed the long-term tolerability of $\mathrm{SC}$ ofatumumab $20 \mathrm{mg}$ in patients who continued treatment with ofatumumab (or were switched from teriflunomide) following completion of the APLIOS, APOLITOS and ASCLEPIOS I/II trials [21]. The association between IgM and IgG levels and the incidence of serious infections was analysed following up to 3.5 years of treatment. The mean serum IgM level was reduced by treatment, but remained above the lower limit of normal (defined as $0.4 \mathrm{~g} / \mathrm{L}$ in the study). The mean serum IgG level remained consistent with baseline values. The risk of serious infections (incidence rate 1.39 per 100 patientyears) was not impacted by changes in IgM or IgG levels [21].

\subsection{Adverse Events of Special Interest}

In the phase III trials, AEs of special interest (AESIs) with ofatumumab included infections, systemic injection-related reactions and malignancy $[6,9,10]$. However, ofatumumab was not associated with an increased frequency of malignancy compared with teriflunomide $(0.5 \%$ vs $0.4 \%$ [6]) in these trials [20], and is not discussed further.

In the pooled ITT populations in both trials, the rate of infections and infestations was $51.6 \%$ in ofatumumab recipients and $52.7 \%$ in teriflunomide recipients [odds ratio 0.96 (95\% CI 0.80-1.15)] [6]. The rate of serious infection (including opportunistic infections such as progressive multifocal leukoencephalopathy, hepatitis B virus reactivation or appendicitis [20]) was $2.5 \%$ and $1.8 \%$ in ofatumumab and teriflunomide recipients [6]. Temporary interruption of treatment or discontinuation due to serious infection occurred in $1.2 \%$ and $0.2 \%$ of patients, respectively [10]. Upper respiratory tract and urinary tract infections were both reported in more than $10 \%$ of ofatumumab recipients (Fig. 2) [20].

Systemic injection-related reactions, most commonly ( $\geq 2 \%$ of patients) fever, headache, myalgia, chills and fatigue $[9,10]$, occurred in more ofatumumab recipients than in teriflunomide recipients who received matching placebo injections (Fig. 2). Most systemic injection-related reactions were mild to moderate in severity and did not require treatment [6]. In ofatumumab recipients, the incidence of systemic injection-related reactions was highest with the first injection $(14.4 \%)$, reducing to $<3 \%$ from the third injection onward $[9,10]$. Two $(0.2 \%)$ grade 3 systemic injectionrelated reactions developed in this group, and ofatumumab was discontinued after the first injection in one patient [6].

\section{Dosage and Administration of Ofatumumab}

Ofatumumab is approved in several countries worldwide, including the USA [9], those in the EU [10] and Japan [11], for the treatment of adults with relapsing forms of MS. In 
the USA, this includes clinically isolated syndrome, relapsing-remitting disease and active secondary progressive disease [9]. In the EU, relapsing MS is defined as active disease characterised by clinical or imaging features [10]. In Japan, ofatumumab can be used for the prevention of recurrence and suppression of progression of physical disorders in patients with relapsing-remitting MS or secondary progressive MS with disease activity [11]. Ofatumumab is available as a $20 \mathrm{mg} / 0.4 \mathrm{~mL}$ pre-filled auto-injector pen (Sensoready ${ }^{\circledR}$ ) or syringe for self-administered SC injection into the abdomen, thigh or outer upper arm $[9,10]$. The first injection of ofatumumab should be administered under the guidance of a healthcare professional. The recommended dosage is $20 \mathrm{mg}$ on days 1,7 and 14 initially, then once monthly from day 28 [9-11].

In the EU, ofatumumab is contraindicated in patients with severe active infection, known active malignancy or who are in a severely immunocompromised state [10]. In the USA, patients with active hepatitis B virus infection must not receive ofatumumab; screening and monitoring for reactivation are advised in at-risk patients [9]. Concomitant use of ofatumumab with immunosuppressant medication (e.g. systemic corticosteroids) may increase the risk of infection [9]; the risk of additive immune system effects must be considered before coadministration $[9,10]$.

As expected with B cell therapy, ofatumumab may decrease IgM levels. In the USA, monitoring of quantitative serum IgM levels is recommended during treatment and after discontinuation of therapy until B cell repletion [9]. Live-attenuated or live vaccines should be administered at least 4 weeks prior to initiation of ofatumumab and, whenever possible, inactivated vaccines should be administered at least 2 weeks prior to initiation of ofatumumab $[9,10]$.

Animal studies indicate that ofatumumab can cause foetal harm (e.g. due to B cell depletion) $[9,10]$ and limited human data suggest that ofatumumab should be avoided during pregnancy [22]. Females of reproductive potential should use effective contraception while being treated with ofatumumab and for 6 months after the last dose [9, 10]. No data are available regarding the use of ofatumumab in breastfeeding women $[9,10]$.

Local prescribing information should be consulted for further detailed information regarding warnings and precautions, vaccinations and use in special populations.

\section{Place of Ofatumumab in the Management of Relapsing Forms of Multiple Sclerosis}

There are many effective DMTs for the treatment of MS, as management is heavily determined by patient response and other patient-specific factors (Sect. 1). In patients with relapsing forms of MS, current guidelines recommend any one of over a dozen different DMTs, including interferons, glatiramer acetate, teriflunomide, dimethyl fumarate, fingolimod, cladribine and the monoclonal antibodies alemtuzumab, natalizumab and ocrelizumab [7, 23]. Another monoclonal antibody, ofatumumab, has the potential to be a useful treatment option for patients with relapsing forms of MS. As the most recent guidelines for MS treatment were published prior to the approval of ofatumumab [7, 23], updates are awaited with interest.

The efficacy and tolerability of SC ofatumumab in adults with relapsing forms of MS were evaluated in two identical phase III trials (Sect. 3). Treatment with ofatumumab resulted in significantly lower annualized relapse rates, MRI-detected lesion activity and disability worsening rates compared with teriflunomide therapy (Sect. 3). Ofatumumab therapy lowered neurofilament light chain levels, but did not lead to a significant change in brain volume; as these are both markers of tissue damage, the discrepancy may require further investigation [6].

Ofatumumab had a generally manageable tolerability profile in phase III trials (Sect. 4). In line with the safety profiles of other anti-CD20 monoclonal antibodies [4], AESIs included infections and systemic injection-related reactions (Sect. 4.1), which were generally manageable with monitoring and/or symptom management $[9,10]$. Infections are a potential safety concern with ofatumumab therapy due to its mechanism of action and based on its prior use in other indications, as well as previous experience with other anti-CD20 monoclonal antibodies [20]. However, there was no significant between-group difference in the incidence of infections in the phase III trials (Sect. 4.1). The risk of infections was also not significantly impacted by the reduced IgM levels resulting from ofatumumab treatment (Sect. 4). Compared with teriflunomide, the frequency of malignancies (an AESI) was not increased by ofatumumab therapy (Sect. 4.1); nevertheless, due to the short duration of these trials and the effect of ofatumumab on the immune system, the potential risk of malignancies developing cannot be excluded [20].

A network meta-analysis (NMA) indirectly compared ofatumumab with other DMTs, as no other direct comparative data are available aside from the ASCLEPIOS trials, and suggests that ofatumumab may be as effective as the monoclonal antibodies alemtuzumab, natalizumab and ocrelizumab [5]. For the endpoints of annualized relapse rate and disability progression, ofatumumab was also found to be superior to or not statistically different from the other nonantibody DMTs (cladribine, dimethyl fumarate, fingolimod, glatiramer acetate, interferon $\beta$ and teriflunomide) analysed. However, given the indirect nature of these comparisons and the lack of long-term clinical outcome data, findings from this analysis should be interpreted with caution [5].

MS is associated with a substantial economic burden, such as through the direct cost of medicines or indirect 
societal impacts from loss of productivity [24]. Ofatumumab was not estimated to be cost effective compared with 10 DMTs (including teriflunomide) from a Canadian healthcare payer perspective [25]. However, the National Institute for Health and Care Excellence (NICE) considers that ofatumumab could be cost effective from a National Health Service perspective [26]. Ofatumumab was estimated to have an incremental cost-effectiveness ratio below the cost effective threshold of $£ 20,000$ per quality-adjusted life-year gained. Although NICE recommends ofatumumab as an effective treatment option in adults with relapsing-remitting MS with active disease (defined by clinical or imaging features) [26], further real-world efficacy and tolerability data (ideally from trials of longer duration) and/or direct comparisons with other DMTs will be useful in determining the place of ofatumumab in the management of relapsing forms of MS.

Although the dosing frequency of some monoclonal antibody treatments may be less frequent (e.g. once or twice per year), ofatumumab has the advantage of being the first targeted B cell therapy that can be self-administered at home (compared with intravenous infusion in a hospital setting), following initial training by a healthcare professional [26]. Both patients with MS and nurses expressed preference for the Sensoready ${ }^{\circledR}$ pen over other auto-injector pens for delivery of their MS therapies, citing ease of use and ability to perform independently [27].

In conclusion, current evidence indicates that ofatumumab is a convenient and effective treatment option, with a generally manageable tolerability profile, for adults with relapsing forms of MS.

\section{Data Selection Ofatumumab: 171 records identified \\ Duplicates removed \\ Excluded during initial screening (e.g. press releases; news reports; not relevant drug/indication; preclinical study; reviews; case reports; not randomized trial) \\ Excluded during writing (e.g. reviews; duplicate data; small patient number; nonrandomized/phase I/II trials) \\ Cited efficacy/tolerability articles

Search Strategy: EMBASE, MEDLINE and PubMed from 1946 to present. Clinical trial registries/databases and websites were also searched for relevant data. Key words were ofatumumab, Kesimpta, multiple sclerosis, RMS, RRMS, CIS, SPMS. Records were limited to those in English language. Searches last updated 16 Nov 2021.
Supplementary Information The online version contains supplementary material available at https://doi.org/10.1007/s40265-021-01650-7.

Acknowledgements During the peer review process, the manufacturer of ofatumumab was also offered an opportunity to review this article. Changes resulting from comments received were made on the basis of scientific and editorial merit.

\section{Declarations}

Funding The preparation of this review was not supported by any external funding.

Authorship and conflict of interest Connie Kang and Hannah Blair are salaried employees of Adis International Ltd/Springer Nature, and declare no relevant conflicts of interest. All authors contributed to the review and are responsible for the article content.

Ethics approval, Consent to participate, Consent for publication, Availability of data and material, Code availability Not applicable.

Open Access This article is licensed under a Creative Commons Attribution-NonCommercial 4.0 International License, which permits any non-commercial use, sharing, adaptation, distribution and reproduction in any medium or format, as long as you give appropriate credit to the original author(s) and the source, provide a link to the Creative Commons licence, and indicate if changes were made. The images or other third party material in this article are included in the article's Creative Commons licence, unless indicated otherwise in a credit line to the material. If material is not included in the article's Creative Commons licence and your intended use is not permitted by statutory regulation or exceeds the permitted use, you will need to obtain permission directly from the copyright holder. To view a copy of this licence, visit http://creativecommons.org/licenses/by-nc/4.0/.

\section{References}

1. Walton C, King R, Rechtman L, et al. Rising prevalence of multiple sclerosis worldwide: insights from the Atlas of MS, third edition. Mult Scler J. 2020;16(14):1816-21.

2. National Institute for Health and Care Excellence. Multiple sclerosis in adults: management. 2019. https://www.nice.org.uk. Accessed 16 Nov 2021.

3. Multiple Sclerosis Coalition. The use of disease-modifying therapies in multiple sclerosis: principles and current evidence. 2019. https://ms-coalition.org. Accessed 16 Nov 2021.

4. Roach CA, Cross AH. Anti-CD20 B cell treatment for relapsing multiple sclerosis. Front Neurol. 2020;11:595547.

5. Samjoo IA, Worthington E, Drudge C, et al. Comparison of ofatumumab and other disease-modifying therapies for relapsing multiple sclerosis: a network meta-analysis. J Comp Eff Res. 2020;9(18):1255-74.

6. Hauser SL, Bar-Or A, Cohen JA, et al. Ofatumumab versus teriflunomide in multiple sclerosis. N Engl J Med. 2020;383(6):546-57.

7. CMSC DMT Guideline Writing Group. The Consortium of Multiple Sclerosis Centers practical guidelines for the selection of disease-modifying therapies in multiple sclerosis. 2019. https:// www.mscare.org. Accessed 16 Nov 2021. 
8. Sanford M, McCormack PL. Ofatumumab. Drugs. 2010;70(8):1013-9.

9. Novartis Pharmaceuticals Corporation. KESIMPTA ${ }^{\circledR}$ (ofatumumab) injection, for subcutaneous use: US prescribing information. 2020. https://www.hcp.novartis.com/products/kesimpta/ rms. Accessed 16 Nov 2021.

10. European Medicines Agency. Kesimpta (ofatumumab): EU summary of product characteristics. 2021. https://www.ema.europa. eu. Accessed 16 Nov 2021.

11. Pharmaceuticals and Medical Devices Agency. Kesimpta subcutaneous injection 20mg pen: Japanese package insert. 2021. https:// www.pmda.go.jp. Accessed 16 Nov 2021.

12. Sorensen PS, Lisby S, Grove R, et al. Safety and efficacy of ofatumumab in relapsing-remitting multiple sclerosis: a phase 2 study. Neurology. 2014;82(7):573-81.

13. Bar-Or A, Wiendl H, Montalban X, et al. Rapid and sustained B-cell depletion with subcutaneous ofatumumab in relapsing multiple sclerosis: APLIOS, a randomized phase-2 study. Mult Scler J. 2021. https://doi.org/10.1177/13524585211044479.

14. Kira JI, Nakahara J, Sazonov DV, et al. Effect of ofatumumab versus placebo in relapsing multiple sclerosis patients from Japan and Russia: phase 2 APOLITOS study. Mult Scler J. 2021. https:// doi.org/10.1177/13524585211055934

15. Bar-Or A, Grove RA, Austin DJ, et al. Subcutaneous ofatumumab in patients with relapsing-remitting multiple sclerosis: the MIRROR study. Neurology. 2018;90(20):e1805-14.

16. Gartner J, Hauser S, Bar-Or A, et al. Benefit-risk of ofatumumab in treatment-naive early relapsing multiple sclerosis patients [abstract no. P0192]. Mult Scler J. 2020;26(Suppl 3):210.

17. Delgado S, Williams MJ, Bagger M, et al. Comparable ofatumumab treatment outcomes in patients across racial/ethnic groups in the ASCLEPIOS I/II and APOLITOS studies [abstract no. 4139 plus poster]. In: 73rd Annual Meeting of the American Academy of Neurology. 2021.

18. Hauser S, Bar-Or A, Cohen JA, et al. B-cell depletion and efficacy outcomes with ofatumumab: subgroup analysis from the pooled phase 3 ASCLEPIOS I and II trials [abstract no. 2356]. Neurology. 2020;94(Suppl 15):2356.
19. Bar-Or A, Schubert-Tennigkeit AA, Mairon N, et al. Dose-dependent tolerability of intravenous and subcutaneous ofatumumab in clinical studies [abstract no. P0316]. Mult Scler J. 2020;26(Suppl 3):272-3.

20. European Medicines Agency. Kesimpta: EU public assessment report. 2021. https://www.ema.europa.eu. Accessed 16 Nov 2021.

21. Jasińska E, Habek M, Wynn D, et al. Impact of ofatumumab on immune responses post-vaccination in RMS patients: ALITHIOS vaccination sub-study design [oral presentation OPR-207]. In: 7th Congress of the European Academy of Neurology. 2021.

22. Hellwig K, Yamout B, Bove R, et al. Pregnancy outcomes in patients with multiple sclerosis following exposure to ofatumumab. In: Consortium of Multiple Sclerosis Centers Annual Meeting. 2021.

23. Montalban X, Gold R, Thompson AJ, et al. ECTRIMS/EAN Guideline on the pharmacological treatment of people with multiple sclerosis. Mult Scler J. 2018;24(2):96-120.

24. Paz-Zuluetaab M, Parás-Bravoa P, Cantarero-Prieto D, et al. A literature review of cost-of-illness studies on the economic burden of multiple sclerosis. Mult Scler Relat Disord. 2020;43:102162.

25. Canadian Agency for Drugs and Technologies. CADTH clinical review report: ofatumumab (Kesimpta). 2021. https://www.cadth. ca/ofatumumab. Accessed 16 Nov 2021.

26. National Institute for Health and Care Excellence. Ofatumumab for treating relapsing multiple sclerosis: technology appraisal guidance [TA699]. 2021. https://www.nice.org.uk/guidance/ta699. Accessed 16 Nov 2021.

27. Ross A, Besser C, Naval S, et al. Patient and nurse preferences for Sensoready ${ }^{\circledR}$ autoinjector pen versus other autoinjectors in multiple sclerosis: results from a multicenter survey [abstract no. P210]. Mult Scler J. 2021;27(Suppl 1):35.

28. Savelieva M, Kahn J, Bagger M, et al. Comparison of the B-cell recovery time following discontinuation of anti-CD20 therapies [abstract no. EP1624]. In: 7th Joint European Committee for Treatment and Research in Multiple Sclerosis-Americas Committee for Treatment and Research in Multiple Sclerosis. 2017. 